\title{
SCIENCES POLITIQUES
}

\section{BENJAMIN CONSTANT \\ ET SA DOCTRINE}

Paul BASTID

Collection "Sciences Politiques", 2 volumes $14 \times 22,5 \mathrm{~cm}$, t. I, 488 pages ; t. II, 628 pages. Les 2 ensemble ......... 85,00 F

THÈSE

\section{HISTOIRE DE LA PRESSE FÉMININE DES ORIGINES A 1848}

Evelyne SULLEROT

Un volume $15,5 \times 24 \mathrm{~cm}, 228$ pages, 16 hors-texte $\ldots \ldots \ldots .42,00 \mathrm{~F}$

\section{Librairie Armand Colin}




\title{
AR GUMENTS Collection dirigée par K̇ostas AXELOS
}

\author{
* \\ Georg Lukacs : Histoire et concience de classe. \\ Kostas Axelos : Marx penseur de la technique. \\ Pierre Broué et Émile Témime : La révolution et la guerre d'Espagne. \\ Carl von Clausewitz: De la guerre. \\ Georges Bataille : L'érotisme. \\ Edgar Morin : Le cinéma ou l'homme imaginaire. \\ François Chatelet : La naissance de l'histoire. \\ Kostas Axelos : Héraclite et la philosophie. \\ Henri Lefebrre : Introduction à la modernité. \\ Harold Rosenberg : La tradition du nouveau. \\ Joseph Gabel : La fausse conscience. \\ B. de Schloezer et M. Scriabine : Problèmes de la musique moderne. \\ Bruce Morrissette : Les romans de Robbe-Grillet. \\ Roman Jakobson : Essais de linguistique générale. \\ Léon Trotsky : De la révolution. \\ Pierre Broué : Le parti bolchevique. \\ Georges Lapassade : L'entrée dans la vie. \\ Herbert Marcuse : Eros et civilisation. \\ Maurice Blanchot : Lautréamont et Sade. \\ G. W. F. Hegel : Propédeutique philosophique. \\ Kostas Axelos : Vers la pensée planétaire. \\ Pierre Fougeyrollas : Contradiction et totalité. \\ Karl Wittfogel : Le despotisme oriental. \\ Karl Korsch : Marxisme et philosophie. \\ Eugen Fink : La philosophie de Nietzsche. \\ Henri Lefebvre : Métaphilosophie. \\ Kudovic Janvier : Pour Samuel Beckett. \\ Louis Hjelmslev : Le langage. \\ Eugen Fink : Le jeu comme symbole du monde. \\ Novalis : Encyclopédie.
}

\section{AUX EDITIONS DE MINUIT}

Rédaction-Administration : 7, rue Bernard-Palissy, Paris-6e. LIT. 39-03. G.G.P. Arguments - Éditions de Minuit. 180-43, Paris.

Abonnement : nouvelle série 1960 ( 64 pages au lieu de 48,4 numéros par an):

Io $\mathrm{F}$; étranger : $\mathrm{I} 5 \mathrm{~F}$; soutien : $20 \mathrm{~F}$.

Directeur-gérant : EDGAR MORIN.

Rédaction : K. Axelos, J. Duvignaud, E. Morin.

Comité : C. Audry, F. Fejtô, P. Fougeyrollas, S. Mallet, D. Mascolo.

Secrétaire de rédaction : REA AxELos.

Le numéro : $3 \mathrm{~F}$

(La diffusion en librairie étant limitée, il est recommandé de s'abonner.) 


\section{COLLECTION U}

Série " Histoire Contemporaine"

\section{LA VIE POLITIQUE EN FRANCE DEPUIS 1789}

par René RÉMOND

\section{HISTOIRE ECONOMIQUE XIX'-XX' SIECLES}

par Jean-Alain LESOURD et Claude GÉRARD

\section{DOCUMENTS D'HISTOIRE CONTEMPORAINE 1776-1963}

par O. VOILLIARD, G. CABOURDIN, F. DREYFUS et R. MARX

LA SOCIÉTÉ FRANÇAISE 1789-1960

par Georges DUPEUX

\section{LA SOCIETÉ SOVIÉTIQUE 1917-1964}

par Pierre SORLIN

LE MONDE CONTEMPORAIN 1945-1964

par Marcel PACAUT et Paul M. BOUJU

\section{LA DECOLONISATION 1919-1963}

par Henri GRIMAL.

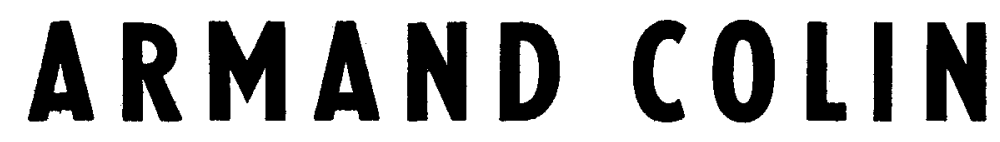




\section{A R C H I V E S \\ E U R O P ÉEN NES DE SOCIOLOGIE}

TOME VII

1966

NUMÉRO I

SOMMAIRE

ALIÉNATION ET STRUCTURE

OR

CONSGIENGE AND CONSGIOUSNESS

MELVIN RICHTER

Intellectual and Class Alienation: Oxford idealist diagnoses and prescriptions.

EUGÈNE FLEISCHMANN

L'esprit humain selon Claude Lévi-Strauss.

EDMUND LEACH

The Legitimacy of Solomon. Some struclural aspects of Old Testament history.

\section{NOTES CRITIQUES}

Peter Berger, Identity as a problem in the sociology of knowledge Serge Moscovici, L'histoire des sciences et la science des historiens WALTER EuchNer, Locke zwischen Hobbes und Hooker. Zu neuen Interpretationen der politischen Philosophie John Lockes.

\section{RÉDAGTION \\ Raymond Aron - Thomas Bottomore - Michel Crozier Ralf Dahrendorf - Eric de Dampierre - Ernest Gellner \\ Musée de l'homme, PARIS-XVIe}

Les Archives paraissent deux fois par an. La correspondance administrative et les abonnements doivent être adressés à la librairie Plon, 8, rue Garancière, Paris-6 ${ }^{\mathrm{e}}$. Le tome, I $8 \mathrm{~F}$; le numéro, $9 \mathrm{~F}$. 


\section{CAHIERS DES ANNALES}

No 20

\section{Recherches sur les douanes à Byzance}

\section{Hélène ANTONIANIS-BIBICOU}

«... une excellente étude, qui apporte une importante et originale contribution à l'histoire des institutions de l'Empire byzantin... »

R. Guilland, Revue des Études Grecques.

« Cet ouvrage d'un auteur spécialisé dans l'histoire byzantine, nous apporte pour la première fois une vue très neuve de l'histoire de l'ensemble des douanes de Byzance. ")

\section{R. Besnier, Revue Économique.}

«... L'auteur fait preuve d'une grande érudition quant au sujet traité, étant également au courant des toutes dernières études des chercheurs occidentaux, de même que soviétiques et bulgares. En conclusion, il convient de souligner une fois de plus que le livre... est une contribution importante et sérieuse... ce livre doit être considéré comme l'une des meilleures réalisations du byzantinisme français contemporain. »

\section{R. Angelov, Études Balkaniques.}

"Hélène Antonianis-Bibicou provides an excellent account of one of basic factors in the Byzantine economy... There is also a good deal of information on details of every life... can still gain insight into social, political and administrative history from this admirable study.

J. Hussey, The English Historical Review.

Cahier $n^{\circ} 20,293$ pages, 2 planches, 3 cartes, 1 carte en pochette................................. $24 \mathrm{~F}$ 


\section{PAST AND PRESENT Revue d'Études Historiques fondée en 1952}

Paraissant en avril, juillet et décembre

« The most valuable historical journals in the western world today are Past and Present and... Annales »

Professor Norman F. CANTOR (1965)

\section{Le fascicule de juillet comprend :}

J. E. SEIGEL ... . . . "Civic Humanism " or Ciceronian Rhetoric? - the Culture of Petrarch and Bruni.

J. F. POUND. . . . . The Social Structure of Norwich, 1525-1575.

NIKKI R. KEDDIE . . Origins of the Religious-Radical Alliance in Iran.

D. R. WATSON . . . The Politics of Educational Reform in France during the Third Republic (1900-1940).

JAME JOLL. .... The 1914 Debate continues- Frit. Fischerand his Critics. ARNO J. MAYOR. . Post-war Nationalisms, 1918-1919.

\section{A paraître bientôt :}

STEFAN KIENIEWICZ. Polish Society and the Insurrection of 1863.

ELENA LOURIE. . . . A Society Organised for War : Medieval Spain.

DENIS TWITCHETT. The Tunhuang Documents and the Study of Chinese Social History.

L. M. CULLEN. . . I Irish History without the Potato.

\section{Ont paru récemment :}

LAWRENCE STONE. . Social Mobility in England, 1500-1700 (avril 1966).

NORMAN STONE. ... Army and Society in the Habsburg Monarchy, 1900-1914 (avril 1966).

T. W. MASON. . . . Labour in the Third Reich, 1933-1939 (avril 1966).

KEITH HOPKINS. . Elite Mobility in the Roman Empire (décembre 1965).

TERENCE RANGER. . Education in East and Central Africa, 1900-1939 (décembre 1965).

V. G. KIERNAN . . . State and Nation in Western Europe (juillet 1965).

T. W. MASON. . . Some Origins of the Second World War (décembre 1964).

Une table des matières complète sera envoyée sur demande.

Tous les numéros épuisés ont été réimprimés

et la série complète est de nouveau disponible.

SOUSCRIPTION : $17 \mathrm{~F}$ par an $(25 \mathrm{~s})$; pour étudiants : $12 \mathrm{~F}$ par an $(15 \mathrm{~s})$.

Numéros déjà parus : $12 \mathrm{~F}$ par exemplaire.

S'adresser au Business Manager : Past and Present

Corpus Christi College, Oxford (Angleterre) 


\section{COMPARATIVE STUDIES IN SOCIETY AND HISTORY}

An International Quarterly

Contents of vol. IX, Number 1 (October 1966)

CLAUDE AKE

E. M. McCLELLAND

GEORGE SHEPPERSON SHEILA KITZINGER

A. H. JOHNS

JAMES R. HOOKER

WOLFRAM FISCHER

JOSEPH R. STRAYER

\section{Charisma}

Charismatic Legitimation and Political Integration.

\section{Religious Movements}

The Experiment in Communal Living in Alyetoro.

Comment.

The Rastafarian Brethren of Jamaica. From Buddhism to Islam: An Interpretation of the Javanese Literature of the Transition.

\section{Social Movements}

Welfare Associations and other Instruments of Accommodation in the Rhodesias between the World Wars.

Social Tensions at Early Stages of Industrialization.

Debate : the role of the military Empires - Some Reflections on Roman and Modern Imperialism.

\section{Review Article}

ARAM A. YENGOYAN Ecological Analysis and Agriculture.

(Any article that is not in itself comparative will be followed by another setting its subject in comparative perspective).

Comparative Studies in Society and History appears four times a year. Annual subscription rate $\$ 6.00$ (U.S.A.) or 23 Dutch guilders (or the equivalent in other currencies), payable through booksellers, through the Editorial Office, Department of History, University of Michigan, Ann Arbor, Michigan, or directly to the publisher : Mouton and Company, The Hague, The Netherlands.

(N.B. Two years' subeription rate $\$ 11.00$ or the equivalent, three year's subscription rate $\$ 15.00$ or the equivalent).

Abonnement : 29,50 F payable chez les libraires ou directement chez l'éditeur: MOUTON of Co, La Haye (Pays-Bas) 


\title{
REVUE \\ TIERS-MONDE
}

\section{CROISSANCE, DEVELOPPEMENT, PROGRES}

\author{
Directeur : François PERROUX
}

Sommaire du Tome VII, no 27, Juillet-Septembre 1966

\section{ARTICLES}

Jean RIOUST de LARGENTAYE : De la liquidité internationale.

Camillo DAGUM : Base et principe pour l'élaboration de modèles en science économique : une approche économétrique.

Anthony BOTTOMLEY : La théorie monétaire de Keynes et les pays en voie de développement.

François PERROUX : Le multiplicateur d'investissement dans les pays sousdéveloppés.

\section{DOCUMENTATION}

Jean BEGUÉ et Moïses IKONICOFF : Sur des techniques de projections dans la programmation du développement économique. Projection de consommation d'acier au Chili.

Jean PONCET : L'expérience des « unités coopératives de production 》 dans la région du Kef.

Yves LA ULAN : Du nouveau dans l'attitude du Fonds Monétaire International envers les pays sous-développés.

Marc GORECKI-LEROY : Réflexions sur la pratique de la prérision de l'emploi dans les pays en voie de développement.

Séminaire Enfance, Jeunesse et Plans de développement. (Paris, 7-18 février 1966).

La Faim dans le Monde : Conférence annuelle du Salut de l'Enfance. (Stockolm. 25 juin 1966).

Compte rendu de la Conférence de Celso Furtado (École Pratique des Hautes Études, Paris, 13 mai 1966) sur l'existence de blocages de développement au Brésil, résultat de l'absorption non planifiée de technologies exogènes.

Robert BADOUIN : Le crédit agricole en Afrique Sud-Saharienne.

\section{BIBLIOGRAPHIE}

DIrection-Rédaction : Institut d'Etude du Développement Economique et Social 58, boulevard Arago, Paris (13\%). Tél. 402-28-01.

Abonnements et ventes: P.U.F., I, place Paul-Painlevé, Paris (5 ). Tél. 033-64-10. Le numéro : $10 \mathrm{~F}$ Abonnement : France et Communauté, $25 \mathrm{~F}$. Etrangér, $30 \mathrm{~F}$. 


\title{
ÉdTIONS DU CENTRE NATIONAL DE LA RECHERCHE SCIENTIFIQUE
}

\author{
I5, quai Anatole-France, PARIS-7•
}

C. C. P. PARIS $9061-11$

Tél. 705-93-39

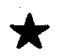

\section{COLLOQUES INTERNATIONAUX DU C.N.R.S.}

\section{Sciences humaines}

\section{LE PROBLÈME DES CAPITALES EN AMÉRIQUE LATINE}

\author{
Toulouse (24-27 février 1964)
}

\begin{abstract}
M. MONBEIG : Réflexions sur les capitales latino-américaines.
M. TRICART : Quelques caractéristiques générales des villes latino-américaines.

M. ROCHEFORT : L'accroissement de la population dans quelques capitales du Brésil.

M. BASTIDE : Ethnologie des capitales latino-américaines.

$M$. POTTIER : La langue des capitales latino-américaines.

$M$. DURAND : Castas y clases en el habla de Lima.

M. MATOS MAR : EI caso del Perú : consideraciones sobre su situacion social como marco de referencia al problema de Lima.

M. JACQUES LAMBERT : Le rôle politique des capitales en Amérique latine.

$M$. BOURRICAUD : La place de Lima dans la vie politique péruvienne.

M. CLAUDE BATAILLON : Mexico, capitale métis.

M. ZAMORANO : Problèmes géographiques de Buenos Aires.

M. VELLAS : Le rôle des capitales dans l'élaboration du Droit international public panamérican.

M. ANGLADE : Une tentative de répartition territoriale du phénomène de la capitale : le municipe brésilien.

M. CANAUX : Le Centre de Recherches d'Urbanisme.

$M$. DENIS LAMBERT : Urbanisation et développement économique en Amérique latine.

M. DOLLFUS : Quelques remarques sur le poids de la capitale dans l'économie péruvienne.

M. ENJALBERT : Les capitales de l'Amérique centrale, sites anciens et essor récent.

M. LASSERRE : Les villes climatiques liées aux capitales de l'Amérique centrale.

M. BOUDEVILLE : Croissance polarisée du Rio Grande do Sul.
\end{abstract}

Symposium sur Brasilia.

Ouvrage in-80 raisin de 408 pages, broché

PRIX : $10 \mathrm{~F}$ 


\title{
GENUS
}

Organe officiel du comité italien pour l'étude des problèmes de la population et de la société italienne de génétique et d'eugénique, sous les auspices du Conseil National des Recherches.

\section{Édité par CORRADO GINI}

Vol. XXI

Année 1965

\section{L'UOMO delle NEVI}

Materiali d'informazione raccolti da

\section{B.F. PORŠNEV}

Dell'Accademia delle Scienze dell'U.R.S.S.

\section{A.A. SMAKOV}

Dell'Università di Mosca

tradotti a cura dell'Associazione ITALIA-U.R.S.S.

\author{
presentati da \\ CORRADO GINI \\ Dell'Università di Roma \\ Presidente del Comitate Internazionale \\ per lo studio degli Ominoidi Villosi.
}

\section{GENUS}

accepte aussi des articles, discours, notes et communications en itallen, français, anglais, allemand et espagnol, dans les différentes sciences ayant trait aux problèmes de la population (Démographie, Sociologie, Économie, Ethnologie, Biologie générale, Génétique, Eugénique, Anthropologie, Hygiène sociale, Psychologie, Géographie).

Prière d'adresser les commandes au Secrétariat du Comité italien pour l'étude des problèmes de la population, via Ombrone, 8/10, Rome, Italia.

Prix du volume (double) : Italie, $\$ 8.000$ - Etranger, U.S.A. $\$ 15$. 


\section{ALETHEIA}

REPREND SA PUBLIGATION

$\mathrm{Au}$ sommaire du $\mathrm{n}^{0}{ }_{4}$ (mai $1966-6,00 \mathrm{~F}$ )

\section{LE STRUGTURALISME}

Avec

Claude Levi-Strauss :

Criteres scientifiques dans les disciplines sociales et humaines.

Entretien avec Roland BarTHEs.

Serge Thion :

Structurologie.

Mauriae Godelier :

Remarques sur les concepts de structure et de contradiction.

Kostas Axelos :

Lucien Sebaq. Entre le marxisme, le freudisme et le structuralisme. et Martin Heidegger :

De l'essence et du concept de Physis, Aristote, Physique B I (III fin).

Note : Georges Lapassade :

«Propédeutique philosophique » de G.W.F. Hegel.

Directeur-gérant : PAUL CAHEN

Rédacteur en Chef : SERGE THION

Rédaction-administration :

22, rue Pernety, PARIS-I $4^{e}$

Abonnements (4 numéros) : France, 20,00 F. Etranger, 25,00 F C.G.P. Paris (Paul Gamen) I3.432-I 7 


\section{Revista de Occidente}

PUBLICACIÓN MENSUAL

INDICE DEL No 39

(JUNIO 1966)

\section{ARTICULOS :}

KARL RAHNER, S. J. : El hombre actual y la religión.

GERMAN BLEIBERG : Mateo Alemán y los galeotes.

RAFAEL SANCHEZ FERLOSIO : Personas $y$ animales en una fiesta de bautizo.

VICENTE SALAS VIU : Luis de Pablo y Cristóbal Halffter.

\section{NOTAS :}

JUAN J. TRIAS VEJARANO : Técnicos y políticos.

PAULINO GARAGORRI : Dos prólogos (de Pedro Laín y José Luis Aranguren a sus obras).

MARIO J. VALDÉS : Observaciones unamunianas.

\section{CRITICA :}

MARIA SCUDERI : Visión conjunta de Machado (recensión de las Obras de A. Machado).

UDO RUKSER : « Der aufbau der Natur », por R. Pannwitz (recensión).

JEAN BÉCARUD : El liberalismo provinciano (recensión de dos novelas de F. García Pavón).

Viñeta de P. PALAZUELO

Número suelto : 50 pts.

Suscripción anual : 500 pts.

Extranjero : 60 pts.

Redacción y Administración : Revista de Occidente S.A.

Bárbara de Braganza, 12

Madrid, 4 (España). Tél. : 231-30-43. 


\section{CAHIERS DES ANNALES}

\section{MICHEL MORINEAU}

\section{JAUGES ET MÉTHODES DE JAUGES ANCIENNES ET MODERNES}

Les termes de marine déroutent et intriguent, tout à la fois, le non-initié. Le plus simple, peut-être, la jauge, n'est pas le moins traître. Que signifie exactement l'unité de jauge employée? Quelle confiance peut-on accorder au tonnage d'un navire? Suivant quelles règles, comparer les bâtiments de mer d'autrefois et d'aujourd'hui ?

Chercher une réponse à ces questions, c'est, bien souvent, s'engager dans des casse-têtes, d'ailleurs passionnants. C'est, surtout, suivre la tentative obstinée des administrateurs et des géomètres pour donner un contour scientifique à une notion qui se révèle inexpugrablement empirique. Et l'un des moindres étonnements n'est pas d'apprendre que la tonne moderne de jauge est, finalement, moins bien définie que le tonneau d'Ordonnance de Colbert...

L'auteur, qui a entrepris cette étude en préliminaire d'une publication sur la flotte française du XVIIe siècle, fournit les équivalences d'un grand nombre d'unités de jauge (ton, tonel, tonneau, tonelada; pipe, botte, salme, last). II renseigne sur les vicissitudes de l'arqueamiento ou jaugeage des navires. Mais sa conclusion reste optimiste car, dans des marges mesurables, les tonnages constituent des matériaux statistiques utilisables et ceci est l'essentiel pour l'histoire économique.

Un volume de 120 pages : $12 \mathrm{~F}$

en vente à la librairie

ARMAND COLIN 


\section{CAHIERS ÉCONOMIQUES ET SOCIAUX}

Vol. IV, n $^{\circ} 2$

Juin 1966

Etude

\section{SOMMAIRE}

H. KNOOP

Some Demographic Characteristics of a Suburban Squatting Community, of Leopoldville

A Preliminary Analysis.

Article

M. VERWILGHEN

Le régime juridique des investissements privés au Congo.

Commentaire du décret-loi du 30 août 1965, portant code des investissements en République Démocratique du Congo.

Notes de recherche

Wyatt Mac GAFFEY

Afterthoughts of Anthropologist : Field

Research in Kongo Central.

\section{Chronique bibliographique}

L'anthropologie et la théorie du changement politique.

M. G. SMITH, Government in Zazzau.

L. A. FALLERS, The King's Men.

J. C. WILLAME.

\section{Documents}

Wyatt Mac GAFFEY, Autobiography of a prophet.

Rédaction, et Administration :

INSTITUT DE RECHERCHES ECONOMIQUES ET SOCIALES

Université Lovanium, Léopoldville XI (Congo)

Abonnement (4 numéros) : CONGO, 1000 F.C.

AUTRES PAYS, 500 F.B. ; U.S. $\$ 10$

AU CONGO : ...... F.C. au compte publications IRES numéro 10.185, SOCOBANQUE, Léopoldville.

HORS CONGO : ...... F.B. ou U.S. $\$$ au compte publications IRES, numéro 3631, Banque Africaine Internationale Bruxelles 5 (Belgique). 
ECOLE PRATIQUE DES HAUTES ETUDES - VI SECTION SCIENCES ECONOMIQUES ET SOCIALES

Collection :

LE MONDE D'OUTRE-MER

PASSÉ ET PRÉSENT

\title{
Gilles SAUTTER
}

\author{
DE \\ L'ATLANTIQUE \\ AU \\ FLEUVE CONGO \\ une géographie du sous-peuplement \\ République du Congo \\ République Gabonaise
}

2 volumes brochés $21 \times 27 \mathrm{~cm}$, I.IO2 pages.......... les $2:$ Igo $\mathrm{F}$ Nombreux graphiques et planches, et cartes hors texte.

\author{
MOUTON \\ 45, RUE DB LILLE \\ PARIS-VII'
}




\title{
Revue Française de Sociologie
}

\author{
publiée par les soìns du \\ CENTRE D'ETUDES SOCIOLOGIQUES
}

avec le concours du

CENTRE NATIONAL DE LA RECHERCHE SCIENTIFIQUE

Directeur : J. STOETZEL

Vol. VII, no 2, Avril-Juin 1966

\section{SOMMAIRE}

Jean STOETZEL......... Les sondages et l'élection présidentielle de 1965.

Pierre NAVILLE ........ Instrumentation audio-visuelle et re-

Jean CAZENEUVE cherche en sociologie.

et Robert PAGES ...... Télévision universitaire et réactions au changement.

Manga BEKOMBO ....... Brazzaville à l'heure de la télévision congolaise.

Renaud SAINSAULIEU... Les classes sociales défavorisées et la télévision.

Georges AUCLAIR ...... Meurtre, inceste et énigme. Étude comparée de presse.

Abraham MOLES ....... Liberté principale, liberté marginale, liberté interstitielle.

INFORMATIONS, BIBLIOGRAPHIE, REVUE DES REVUES, RÉSUMÉS DES ARTICLES EN ANGLAIS, ALLEMAND, ESPAGNOL, RUSSE.

Direction, Rédaction :

CENTRE D'ÉTUDES SOCIOLOGIQUES

82, rue Cardinet, Paris (170)
Administration, abonnements : ÉDITIONS DU C.N.R.S. 15, quai Anatole-France, Paris $\left(7^{\circ}\right)$

5 numéros de 144 pages et un numéro spécial

ABONNEMENT : FRANCE........... 30 Francs (le numéro : 7 F)

ETRANGER .......... 30 -

U. S. A............ \$ $\$ 6,00$

C.C.P. Paris 9061-11. Centre National de la Recherche Scientifique (Service des Publications) 


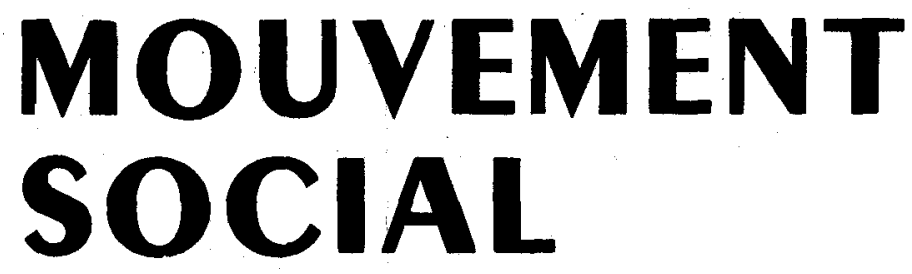

Revue trimestrielle de

I'INSTITUT FRANÇAIS d'HISTOIRE SOCIALE (Association reconnue d'utilité publique)

Publiée avec le concours du C.N.R.S.

\section{Études :} SOMMAIRE

Associations et syndicalismes universitaires, 1828-1928, par P. GERBOD.

Les professeurs du second degré au début du $X X \cdot$ siècle, par G. VINCENT.

\section{Travaux Universitaires :}

Juin 1936 à Caen, par G. LEMARCHAND.

La grève du 30 novembre 1938, par G. BOURDÉ.

Élections professionnelles, par M. PERROT et J. COMMAILLE.

Notes critiques :

La Revisione del marxismo in Italia, par E. SANTARELLI (R. Paris). - Pouvoir et société économique, par J. LHOMME (J. Bouvier). - The Trotsky Papers, 1917-1922. Première partie, 1917-1919 (J. Dautry). - Histoire du Front Populaire, 19341938, par G. LEFRANC (J. Maitron). - Histoire de l'Algérie contemporaine : I. - La conquête et les débuts de la colonisation, 1827-1871, par Ch.-A. JULIEN ; L'Algérie : nation et société, par M. LACHERAF ; La vie musulmane en Algérie d'après la jurisprudence de la première moitié du $X X \cdot$ siècle, par J.-P. CHARNAY (M. Rodinson).

Notes de lecture :

Textes choisis de R. OWEN (J. Gans). - Frédéric Degeorge, par A. FORTIN (J. Maitron). - El Movimento obrero en España. La Primera Internacional, 1864-1881, par J. TERMES ARDEVOL (R. Lamberet). - L'Internationale de 1864. Libres propos, par HEM DAY (J. Maitron). - Le patronat européen du Maroc, action sociale, action politique, 19311942, par R. GALLISSOT (J. Bouvier). - Giornali fuori legge : la stampa clandestina antifascista, 1922-1943, par A. DAL PONT, A. LEONETTI, M. MASSARA (R. Paris).

Archives :

Les origines du socialisme en Ariège, par R. GARMY.

\section{LES EDITIONS OUVRIERES}

12, avenue de la Sœur-Rosalie, PARIS-XIIIe Abonnement : France, $20 \mathrm{~F}$; Ettranger, $25 \mathrm{~F}$ (4 numéros)

C.C.P. Paris 1360-14 


\section{GAUTHIER-VILLARS - ÉDITEUR - PARIS}

vous recommande ses trois nouvelles collections

\section{SCIENCES HUMAINES APPLIQUEES \\ dirigée par Paul ALBOU}

Diverses par les domaines qu'elles explorent, inégalement assurées de leurs résultats mais toujours critiques à l'égard des techniques qu'elles utilisent, les Sciences Fumaines Appliqúées constituent aujourd'hui un secteur particulièrement dynamique de la connaissance scientifique, ou la théorie ne renie plus la pratique qui la suscite et qu'elle éclaire en la perfectionnant. La collection « Sciences Humaines Appliquées 》 se propose de démontrer qu'une telle diversite, loin de nuire aux progrès de cette connaissance, contribue à la réussite de programmes d'intervention qui visent, tout à la fois, à comprendre le monde, mais aussi à le transformer.

Vient de parâttre :

PASTEURS-NOMADES MEDITERRANEENS (Les Saracatsans de Grèce), par Georges B. Kavadias, Docteur ès Lettres, 444 pages, 109 illustrations, 1965. Cartonné. . . . . 50 F

\section{MATHEMATIQUES ET SCIENCES DE L'HOMME}

\section{Collection publiêe par le Centre de Mathématique Sociale et de Statistique} Ecole pratique des Hautes Études

La collection se propose de publier des ouvrages originaux ou des traductions, sur les techniques d'origine mathématique utilisées dans les Sciences de l'Homme; ce qui entraíne la publication d'ouvrages proprement mathématiques concernant les thèmes universels dont l'intervention est nécessaire dans toute formalisation.

La collection s'adresse aux chercheurs, aux enseignants et aux étudiants en Sciences de l'Homme qui utilisent des instruments mathématiques.

Récemment parus :

L'ANALYSE HIÉRARCHIQUE, par Benjamin Matalon, 150 pages . . . . . . . . . . $25 \mathrm{~F}$ THEORIE DES GRAPHES ET STRUCTURES SOGIALES, par Claude Flament, 166 pages ....... 35 F En coédition avec MOUTON et Gie, PARIS, LA HAYE

\section{HOMMES ET ORGANISATIONS dirigée par Jean ARDOINO}

Dans le vaste creuset où s'opèrent les métamorphoses sociales, quelle est, et que demerure, la place de l'homme ? Quelles sont les principales maladies sociales contemporaines? Quels peuvent en être les remèdes? Quelles peuvent être les ressources techniques et humaines pour faciliter l'adaptation de l'homme, tant à l'échelon des individus qu'à celui des groupes ot des organisations? Tels sont les domaines privilégiés auxquels la collection « Hommes et Organisations 》 se propose d'apporter sa contribution.

Récemment parus :

PATHOLOGIE SOCIALE DE L'ENTREPRISE, par Robert MeIGNIEz, 228 pages . . . . . . . . . . . . 22 F PROPOS ACTUELS SUR L'EDUCATION, par Jean ARdoivo, 304 pages $45 \mathrm{~F}$ 


\section{LA TABLE RONDE \\ $N^{o}$ 222-223 - Juillet-Août 1966}

Nouvelles :

Ana-Maria MATUTE : Le Maître.

François NÉRAULT : La bouée.

Michèle SAINT-LO : Week-end.

Ernst WIECHERT : Le buisson ardent.

Poèmes de Michel BAYAT, Philippe CHABANEIX, Thomas MERTON et André PIETTRE.

Débat sur Au hasard, Balthazar de Robert BRESSON, entre M. Michel ESTEVE, M. Stanislas FUMET, Mme Mireille LATILLE DANTEC et M. Jean D'YVOIRE.

\section{Chroniques :}

Jacques de BOURBON BUSSET : Séquences.

Henri GOUHIER : Le Théâtre'des Nations (Banlieue et petites scènes).

Jacques GUILLERME : Entre Seine et Vlatava.

Paul WERRIE : Le roman qui se fait de ne pas se faire.

Isabelle ESMEIN : La vie littéraire et artistique en U.R.S.S. et le XXIII Congrès du Parti.

Notes sur livres français et étrangers.

Livres d'art.

\section{$\star$ \\ S. E. P. A. L.}

23, rue du Renard, Paris-IVe - TUR. 16-39

C.G.P. 16 217-25

Abonnement : FRANCE ...... 1 an, $42 \mathrm{~F}$; 6 mois, $21 \mathrm{~F}$ ETRANGER ... 1 an, $50 \mathrm{~F}$; 6 mois, $25 \mathrm{~F}$ 


\title{
TECHNIQUES ÉCONOMIQUES MODERNES
}

Collection publiée sous la direction d'André PIATIER

SÉRIE PRODUCTION ET MARCHÉS, 5

\section{L'IMPORTANCE ÉCONOMIQUE \\ DE LA MARQUE}

\author{
par C.-H. GIRAUD
}

PIATIER A. et collaborateurs. - Les formes modernes de la concurrence,

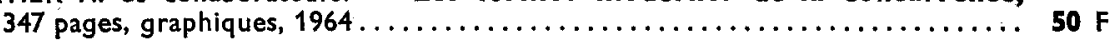

AVRIL P. - Théorie sommaire de la distribution des biens de consommation.

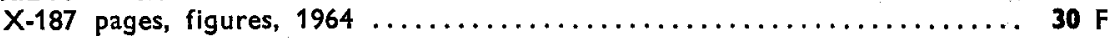

HENON R. - L'Econométrie au service de l'entreprise. XXIl-478 pages, figures, 1964

QUIN Cl. - Physionomie et perspective d'évolution de l'appareil commercial français (1950-1970). XII-364 pages, 1964 .

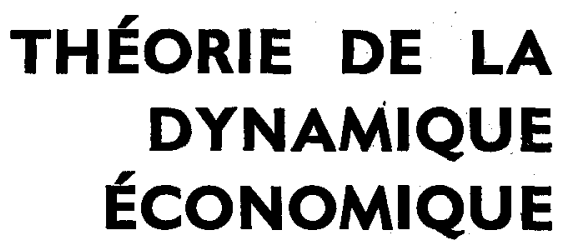

Essai sur les variations cycliques et à long terme de l'économie capitaliste par M. KALECKI

D'après la 2e édition anglaise. Traduite et préfacée par M. LUTFALLA

LUTFALLA M. - L'état stationnaire. VIII-371 pages, $1964 \ldots \ldots \ldots \ldots \ldots \ldots \ldots 48$ F

SHISKIN J. - Les signaux avertisseurs des récessions et des reprises. XVI203 pages, 1964

SAMUELSON R. A. - Les fondements de l'analyse économique. 525 pages, 1965. $80 \mathrm{~F}$ HELINE R. - Étude méthodologique du capital national. 417 pages, 1965 .... 86 F

\section{GAUTHIER-VILLARS Éditeur PARIS}




\section{CRITIQUE REVUE générale des publications FRANÇAISES ET ÉTRANGËRES \\ Direction-rédaction : Jean PIEL}

No 229 (juin 1966)

\section{Numéro consacré à MAURIGE BLANGHOT}

René CHAR. - Conversation avec une grappe, en hommage à Maurice Blanchot.

Georges POULET. - Maurice Blanchot, critique et romancier.

Jean STAROBINSKI. - Thomas l'obscur.

Emmanuel LEVINAS. - La servante et son maître.

Michel FOUCAULT. - La pensée du dehors.

Paul DE MAN. - La circularité de l'interprétation dans l'œuvre critique de Maurice Blanchot.

Francoise COLIN. - L'un et l'autre.

Roger LAPORTE. - Le oui, le non, le neutre.

Jean PFEIFFER. - La passion de l'imaginaire.

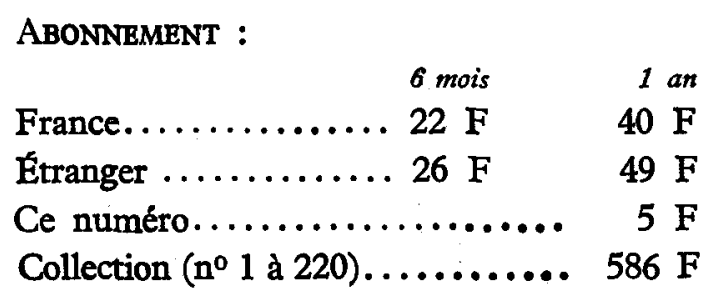

\section{AUX EDITIONS DE MINUIT}

7, rue Bernard-Palissy, Paris-vi ${ }^{\mathrm{e}}$

(C.C.P. Paris 180-43) 


\title{
REVUE ECONOMIQUE
}

\author{
publication bimestrielle \\ avec le concours de la VI. Section de l'École Pratique des Hautes Études \\ et du Centre National de la Recherche Scientifique
}

Vol. XVII

No 4 - Juillet 1966

P.ELAU La rationalité du taux d'actualisation.

P. H. DERYCKE Taux d'intérêt, taux d'actualisation et croissance économique.

J. BOURRINET Note sur l'afflux des investissements directs dans la C.E.E.

J. F. BESSON Centralisation et décentralisation : le problème des biens collectifs.

R. SOURDAIN La courbe d'offre individuelle de travail.

M. LUTFALLA Economistes de jadis : L'œurre économique de Maurice Bourguin.

J. WEILLER Chronique : L'économie internationale du $\mathrm{XX}$ siècle et les analyses de politique économique.

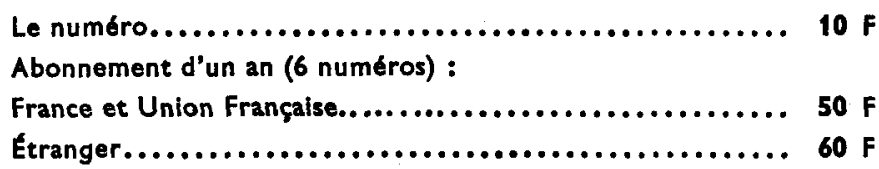

\section{LIBRAIRIE ARMAND COLIN}

103, boulevard Saint-Michel, PARIS-Ve

C.C.P. PARIS 21-335-25 


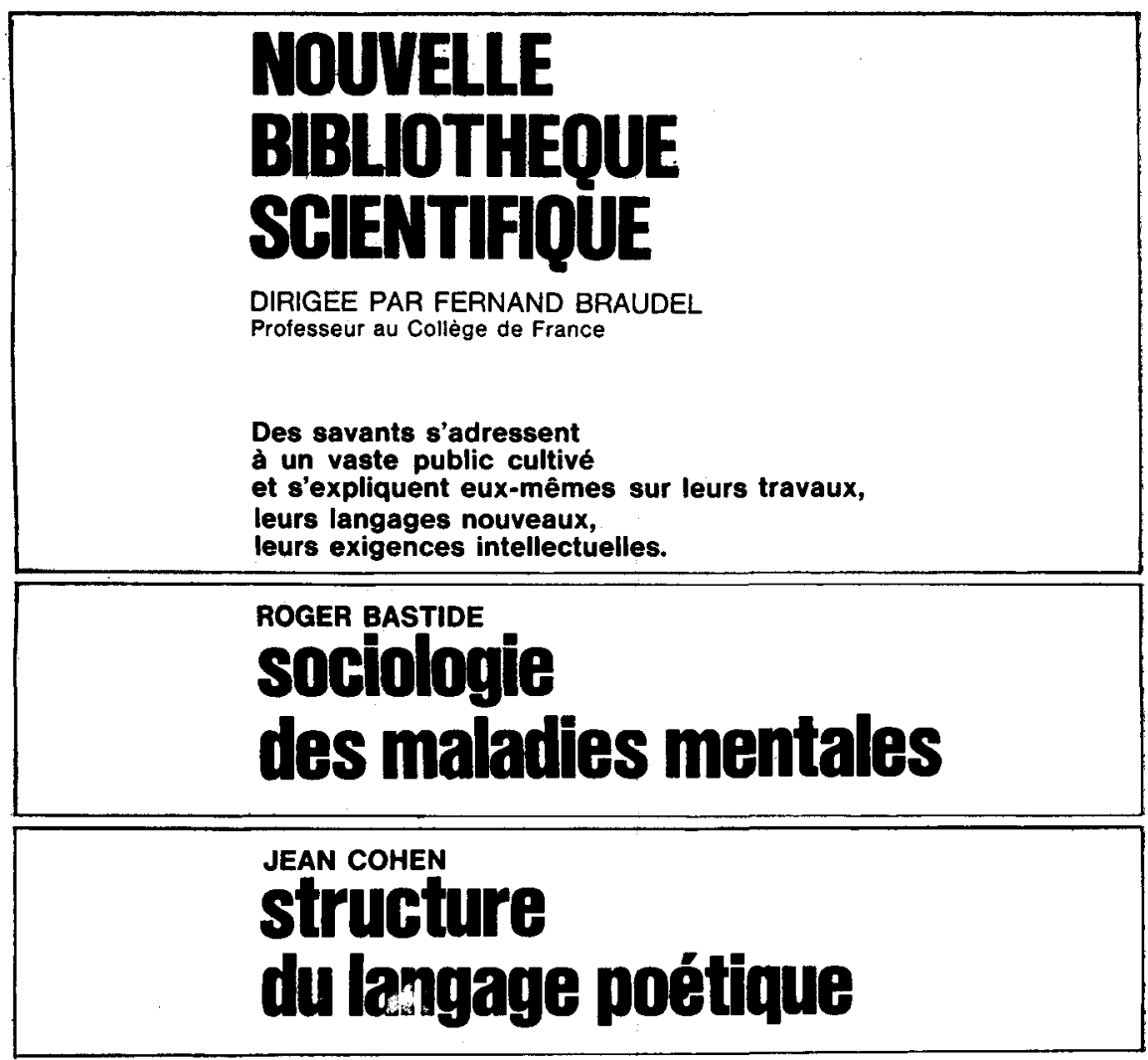

FRANCYIS NEWTON

une sociologie du jazz

SYLVAIN WICKHAM

concentration et dimensions de l'entreprise

ROBERT WALLIS

8 GIIIST vient de paraitre

quatrième dimension

de l'esprit

\section{flammarion}

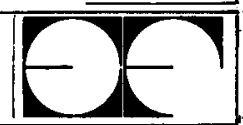




\title{
ANNALES DE NORMANDIE
}

Revue trimestrielle d'Etudes régionales

Rédaction et administration :

Logls des Gouverneurs - Château de Caen

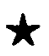

Sommaire du $n^{\circ} 2$, juin 1966 (XVI année)

J.-C. GÉGOT, Étude par sondage de la criminalité dans le bailliage de Falaise (XVIIe-XVIIIe siècles). Criminalité diffuse ou société criminelle?

J.-J. BERTAUX, Jeux traditionnels. Le jeu de bouchon ou de palets.

\author{
CHRONIQUE DES ÉTUDES NORMANDES \\ (Facultés et Écoles - Bulletin critique)
}

Les Annales de Normondie publient chaque année, en mars, conjointement avec les Cahiers Léopold Delisle, une bibliographie des travaux concernant la Normandie parus durant l'année précédente; un autre numéro est consacré désormais, chaque année, à l'archéologie.

\section{Abonnement 1966:}

- France, $12 \mathrm{~F}$; Etranger, $15 \mathrm{~F}$.

- Le numéro archéologique, $8 \mathrm{~F}$.

A verser à la Société d'Enquêtes Ethnographiques Normandes, Logis des Gouverneurs, Château de Caen; C. C. P. Rouen 604-01. 


\section{ANNALES \\ ECONOMIES - SOCIÉTÉS - CIVILISATIONS \\ Rédaction : 20, rue de La Baume, Paris-VIIIe \\ Administration : LIBRAIRIE ARMAND COLIN, 103, Bd St-Michel, PARIS-V• \\ Compte de chèques postaux : Parig, $N \circ 21335-25$}

Abonnement 1965 (6 numéros)

France, Communauté française, Maroc, Tunisie, Guinée. . . . . . . . . . . . . . . 29 F

Autres pays. . . . . . . . . . . . . . . . . . . . . . . . . . 35 F

Le numéro de 208 pages. . . . . . . . . . . . . . . . . . . . . . . . . . . 6 F

Années parues. . . . . . . . . . . . . . . . . . . . . . . . . . . . . 20,F

\section{CAHIERS DES ANNALES}

1. P. Sardella, Nouvelles et spéculations à Venise au début du xvie siècle...................... $2,90 \mathrm{~F}$

2. Ch. Moraze, Trois essais sur Histoire et Culture.... 2,40 F

3. Marc Bloch, Apologie pour 1'Histoire ou Métier d'Histo-

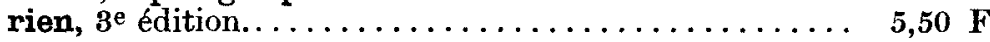

4. A travers les Amériques latines (Recueil publié sous la direction de Lucien Febvre)..................

5. Georges Friedmann, Humanisme du Travail et Humanités. ..................... Epuisé.

6. L. E. Halk IN, Initiation à la critique historique....... 15,00 F

7. G. Debien, En haut Poitou : défricheurs au travail (xve

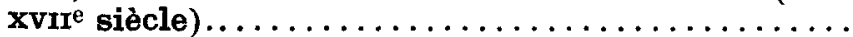

8. A. Tenenti, La Vie et la Mort à travers l'art du xve siècle

9. Marc Bloch, Esquisse d'une histoire monétaire de l'Europe

10. A. KoYré, Mystiques, Spirituels, Alchimistes du xvie

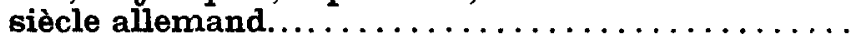

11. G. Debien, Etudes antillaises au Xvirre siècle.

$3,75 \mathrm{~F}$

$5,30 \mathrm{~F}$

$3,25 \mathrm{~F}$

$4,80 \mathrm{~F}$

$7,00 \mathrm{~F}$

12. F. L'Huilliter, La lutte ouvrière à la fin du Second Empire.

13. Marc Bloch, La France sous les derniers Gapétiens

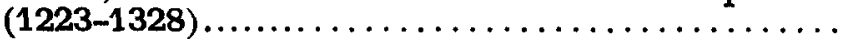

14. Maurice Dommanget, A. Blanqui, et l'opposition révolutionnaire à la fin du Second Empire...............
Introduction à la Révolution française, pré-

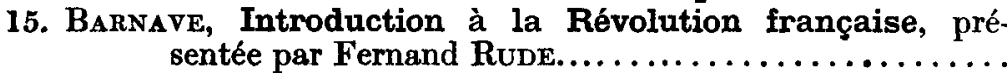

16. Marc Bloch, Seigneurie française et manoir anglais...

17. M. Delafosse et Cl. Laveau, Le Commerce du sel de Brouage aux xvire et $\mathrm{xvIrr}^{\mathrm{e}}$ siecles..............

18. A. Daumard et F. Furet, Structures et relations sociales à Paris au milieu du xvıre siecle............. $8,00 \mathrm{~F}$

19. A. Koyré, Études d'histoire de la pensée philosophique. 15,00 F

20. H. Antoniadis-Bibicov, Recherches sur les douanes à Byzance......................... 24,00 F

21. H. Hauser, La modernité du xvie siècle...........9,80 F

22. Thomas Plat ter, Autobiographie (texte traduit et présenté par Marie Helmer) .................... 11,00 F

23. Louis Dumont, La civilisation indienne et nous. Esquisse de sociologie comparée .................. 10,00 F

24. Michel Mor inead, Jauges et méthodes de jauges anciennes et modernes................................. 12,00 F 


\section{témoignage d'un historien}

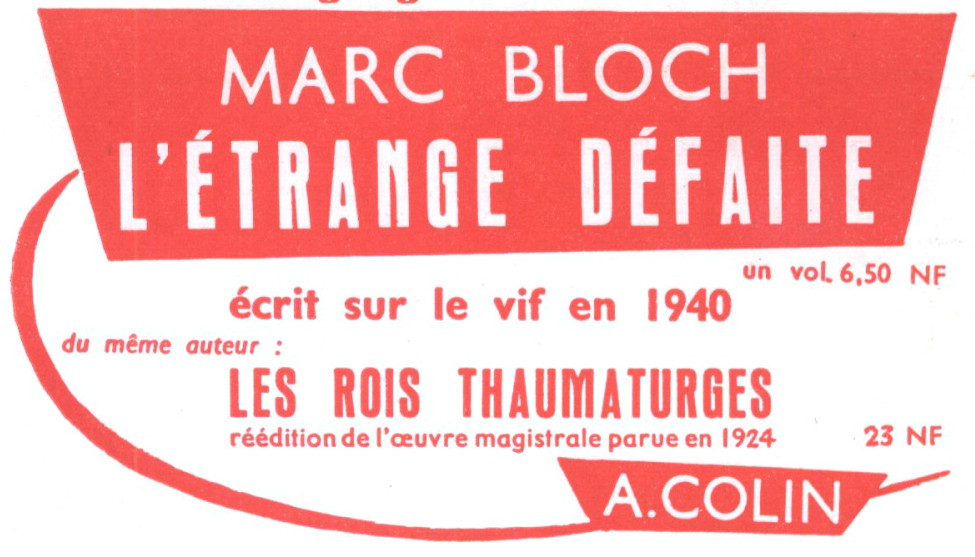

Ce qu'en pense la critique :

« Marc Bloch a été, entre les deux guerres mondiales, un de nos meilleurs historiens. Il fut aussi un martyr de la Résistance, au sens fort du mot martyr : en étant torturé et tué... Tout ce que rapporte Marc Bloch avec tant de justesse et de modestie sur les défaillances de l'armée française est vrai, j'en suis persuadé. »

Emmanuel Berl

(Preuves)

"Son livre est donc, d'abord, un témoignage d'autant plus précieux qu'il émane d'un des plus grands, des plus authentiques historiens que la France ait jamais produits. ")

Henri Noguères

(Aux Carrefours de l'Histoire)

«De tous ses ouvrages celui qui, humainement, est sans conteste le plus remarquable, celui qui devrait toucher non seulement les spécialistes, mais le grand public : c'est L'étrange défaite, ce journal de guerre, cette méditation, dont la lucidité, les analyses psychologiques, les anecdotes caractéristiques, les rapprochements entre le passé et le présent font le type même de l'ouvrage de chevet, auquel on revient sans cesse pour relancer sa propre réflexion. "

HENRI BRUNSGHWIG (O.R.T.F.) 